\title{
Construction of an artificial recombinant bicistronic plasmid DNA vaccine against porcine rotavirus
}

\begin{abstract}
Authors:
Tingting Cui

Jun Xiong ${ }^{1}$

Yongzhi Wang

Xintian Wen ${ }^{1}$

Xiaobo Huang ${ }^{1}$

Yong Huang ${ }^{1}$

Xiaoping $\mathrm{Ma}^{1}$

Zhongkai Hu${ }^{1}$

Qin $\mathrm{Zhao}^{3}$

Sanjie $\mathrm{CaO}^{1}$
\end{abstract}

Affiliations:

${ }^{1}$ College of Veterinary

Medicine and Institute

of Preventive Veterinary

Medicine, Sichuan

Agricultural University, China

${ }^{2}$ Sichuan Rural Science and Technology Development Centre, Chengdu, China

${ }^{3}$ College of Food Science, Sichuan Agricultural

University, China

Correspondence to:

Sanjie Cao

Email:

csanjie@gmail.com

Postal address:

46 Xinkang Road, Ya'an

625014, China

Dates:

Received: 27 June 2012

Accepted: 31 Oct. 2012

Published: 04 Mar. 2013

How to cite this article:

Cui, T., Xiong, J., Wang, Y.,

Wen, X., Huang, X., Huang,

Y. et al., 2013, 'Construction

of an artificial recombinant

bicistronic plasmid DNA

vaccine against porcine

rotavirus', Onderstepoort

Journal of Veterinary

Research 80(1), Art. \#498,

8 pages. http://dx.doi.

org/10.4102/ojvr.v80i1.498

\section{Copyright:}

(C) 2013. The Authors.

Licensee: AOSIS

OpenJournals. This work

is licensed under the

Creative Commons

Attribution License.

\section{Read online:}

The attenuated Salmonella typhimurium $\chi 4550$ strain was used to harbour a reconstructed bicistronic DNA vaccine against porcine rotavirus, which carried the rotavirus nonstructural protein 4 (NSP4) and VP7 genes simultaneously. Using a balanced lethal system, the kanamycin resistance gene of expressing eukaryotic plasmids $\mathrm{PVAX} 1$ and $\mathrm{pVAXD}$ were replaced by the aspartate $\beta$-semialdehyde dehydrogenase (asd) gene. The NSP4 cleavage product (259-525) of rotavirus OSU strain and VP7 full-length genes were amplified by reverse transcription polymerase chain reaction and then inserted into the eukaryotic single-expression plasmid, pVAX1-asd, and the eukaryotic dual-expression plasmid, pVAXD-asd, respectively. The recombinant plasmids pVAX1-asd-NSP4, pVAX1-asd-VP7 and pVAXD-asd-NSP4-VP7 were transformed into the attenuated $S$. typhimurium $\chi 4550$ strain by electrotransformation. An indirect immunofluorescence assay of the expressed COS-7 cell suggested that the recombinant S. typhimurium $\chi 4550$ strain was constructed successfully. The recombinant S. typhimurium $\chi 4550$ strain was orally administered to BALB/c mice. The group immunised with dualexpression plasmids produced a significantly higher level of serum Immunoglobulin G (IgG) and intestinal Immunoglobulin A (IgA) than the group immunised with single-expression plasmids. These results indicated that eukaryotic bicistronic plasmid DNA vaccines could be successfully constructed to enhance humoural, mucosal and cellular immune response against rotavirus infection.

\section{Introduction}

Rotavirus is a highly infectious, triple-layered icosahedral, non-enveloped virus particle with a genome of 11 segments of double-stranded Ribonucleic acid (RNA) (Petrie, Estes \& Graham 1983). It is recognised as the most important cause of acute gastroenteritis and dehydration in young children and animals (Estes 2001). Although highly effective, currently available reassortant tetravalent rhesus rotavirus vaccines were found to cause a higher occurrence of intussusception in young children (Glass et al. 2006). Moreover, the safe rotavirus-like particle (VLP) vaccines induce only partial protection and intestinal antibody secreting cell responses when used in conjunction with live attenuated human rotavirus (Gonzalez et al. 2004; Yuan et al. 2001) and 2/6-VLP vaccines do not protect suckling mouse pups from rotavirus diarrhoea (Coste et al. 2000). Thus, effective protective rotavirus vaccines that are unlikely to cause intussusception would be highly desirable. The use of DNA vaccines is a new approach to protect animals and children against rotavirus. VLP and DNA vaccines may constitute a third generation of rotavirus vaccines (Chen et al. 1998; O'Neal et al. 1997). It was previously reported that parenteral immunisation with rotavirus VP4, VP6 and VP7 DNA vaccines induce high levels of serum rotavirus Immunoglobulin G (IgG) but fail to protect mice against viral challenge (Chen et al. 1997; Herrmann et al. 1999; Yang et al. 2001). It is therefore necessary to develop an efficient method to increase the immunogenicity of DNA vaccines.

The rotavirus nonstructural protein 4 (NSP4) is the first described viral enterotoxin and may inducing dose- and age-dependent diarrhoea in neonatal mice without causing histological alterations (Ball et al. 1996). When mice were infected with rotavirus, NSP4-specific Immunoglobulin A (IgA) antibodies were generated in response to mucosal immunisation and transcytosed from the basal lamina into the mucosal epithelium (Gebert 1997). Further, orally immunised mice produced maternal antibodies against NSP4, generating passive protection in newborn mouse pups against rotavirus challenge (Ward, Rich \& Besser 1996). Thus, NSP4 is thought to be a promising candidate for a rotavirus DNA vaccine that induces a significant and effective immune response (Ball et al. 2005).

VP7 is a structural protein of the outer capsid of a rotavirus and is encoded in the seventh, eighth or ninth gene segment of the genome (depending on the viral strain) (Song \& Hao 2009). VP7 may be the dominant immunogen for the production of neutralising antibodies, which are thought to have an important role in the protection against rotavirus-induced diarrhoea (Ward et al. 1990, 1993) and associate resistance to protect the host against diarrhoea upon challenge with the virulent 
parental rotaviruses (Hoshino et al. 1988). Matsui et al. (1989) demonstrated that VP7 monoclonal antibodies (MAbs) have two distinct epitopes, with the specific epitopes defined by VP7-specific MAb 57-8 mediating protection against at least two rotavirus serotypes. From this we concluded that VP7 plays an important role in resisting rotavirus challenge.

In this study, a bicistronic plasmid was formed as a coexpression vector carrying the C-terminal of the porcine rotavirus (PRV) NSP4 and VP7 genes, and then inserted into the Salmonella typhimurium $\chi 4550$ strain as a DNA vaccine that was orally administered to BALB/c mice. The S. typhimurium $\chi 4550$ strain consists of attenuated bacteria of which the asd gene has been deleted. We evaluated the mucosal and systemic antibody responses. The results showed that the coexpression DNA vaccine mediated by a bicistronic plasmid could significantly enhance humoural and mucosal immune response.

\section{Materials and method}

\section{Bacterial strains, plasmid, virus, cell lines and experiment mice}

The attenuated S. typhimurium $\chi 6212, \chi 3730$ and $\chi 4550$ strains and plasmid PYA3342 were kindly provided by Professor Kai Schulze of the Helmholtz Centre for Infection Research, Braunschweig, Germany. The eukaryotic expression vector pVAX1 was purchased from Invitrogen, Carlsbad. It 'contains cytomegalovirus (CMV) immediate-early promoter for efficient expression and bovine growth hormone (BGH) poly A signal for mRNA stability' (Yang et al. 2009). The OSU rotavirus strain was used to generate cDNA and produce virus antigen stock, which was applied as the antigen in the enzyme-linked immunosorbent assays (ELISAs). MA104 cells and COS-7 cells were purchased from the China Center for Type Culture Collection (Wuhan, China). Six-weekold inbred BALB/c female mice were obtained from the Chengdu Institute of Biological Products (Chengdu, China).

\section{Construction and reforming of the eukaryotic bicistronic expression vector pVAXD-asd}

The vectors pVAXA and PVAXB were constructed by reforming the multiple cloning site of $\mathrm{pVAX} 1$. The product of pVAXA polymerase chain reaction (PCR) (-PCMVMCS-BGHpolyA-) was linked with $\mathrm{pVAXB}$ to form vector pVAXD with two different multiple cloning sites. Dualenzyme digestion identification showed that the bicistronic vector pVAXD was successfully constructed. The aspartate $\beta$-semialdehyde dehydrogenase (asd) gene was amplified by PCR and digested by restriction enzymes BamHI and XbaI from plasmid PYA3342. We then used the Klenow fragment enzyme character to fill the sticky end of DNA fragments derived by enzyme restriction digestion to a flat end, and linked it with $\mathrm{pVAX} 1$ and $\mathrm{pVAXD}$ vectors that were removed from the 5 ' terminal phosphate group by calf intestinal alkaline phosphatase. The reformed plasmids, pVAX1-asd and pVAXD-asd, were reconstructed successfully and plasmid pVAXD-asd was sequenced by Invitrogen, Shanghai.

\section{Construction of vaccine plasmid and its transformation into attenuated Salmonella typhimurium}

Viral RNA was extracted from MA104 cells infected with the OSU rotavirus strain by using the RNAsimple Total RNA Kit (TIANGEN, Beijing). Total cDNA was obtained from the viral total RNA by a reverse transcriptase reaction procedure. The NSP4 segment was amplified from the cDNA using the sense primer S1 5'-CTGCAGCCGCCATGGAACAGGTTACTAC-3' (forward) and the antisense primer 5'-CTCGAGCTCACATA GACGCAGTTACTTCCGAC-3' (reverse). Underlined areas indicate the PstI and XhoI sites, respectively. The VP7 segment was amplified from the cDNA using the sense primer S2 5'-GCTAGCCGCCATGTATGGTATTGAAT-3' (forward) and the antisense primer 5'-GGTACCATCTAGACTCGGT AATAAAAGGCAG-3' (reverse). Underlined areas indicate the NheI and KpnI sites, respectively. The amplified DNA fragments of the NSP4 and VP7 genes were cloned into the pMD19-T vector (TaKaRa) and sequenced. The NSP4 fragment was released from the pMD19-T plasmid by PstI and $\mathrm{XhoI}$ digestion and ligated into the reformed pVAX1-asd plasmid. pVAXD-asd vectors were cut by the same enzymes. The recombinant plasmids were named pVAX1-asd-NSP4 and pVAXD-asd-NSP4. The VP7 fragment was subsequently released from the pMD19-T plasmid by NheI and KpnI digestion and ligated into the pVAX1-asd and pVAXD-asdNSP4 vectors, cut by the same enzymes. The recombinant plasmids were named pVAX1-asd-VP7 and pVAXD-asdNSP4-VP7. These plasmids, including pVAX1-asd-VP7, pVAX1-asd-NSP4, pVAXD-asd-NSP4-VP7 and pVAX1-asd, were electroporated into $S$. typhimurium $\chi 3730$ with a cuvette $(1.8 \mathrm{kV}, 200 \Omega, 25 \mu \mathrm{F})$.

Positive transformants were electroporated into S. typhimurium $\chi 4550$ using the same method as described above. Lastly, the positive transformants were verified by PCR amplification and digested with restriction enzymes. The resulting strains were named $\chi 4550$ (pVAX1-asd-VP7), $\chi 4550$ (pVAX1-asd-NSP4), $\chi 4550$ (pVAXD-asd-NSP4-VP7) and $\chi 4550$ (pVAX1-asd).

\section{Transfection of COS-7 cells and transient expression}

COS-7 cells were seeded in six wells of tissue culture plates (Costar, Shanghai). Cells were transfected with the recombinant plasmids pVAX1-asd-VP7 in the first well, pVAX1-asd-NSP4 in the second well, pVAXD-asd-NSP4-VP7 in the third and fourth wells, and pVAX1-asd in the fifth and sixth wells according to the instructed protocol provided by the supplier (Invitrogen Life Technologies, Carlsbad). Cells were washed with phosphate buffered saline (PBS) after 36 hours, then fixed with an ice-cold methanol and acetone mixture $(1: 1)$ at $4{ }^{\circ} \mathrm{C}$ for $30 \mathrm{~min}$ and washed again with PBS. Two kinds of diluted primary antibody were collected from rabbits immunised by prokaryotic express protein VP7 and NSP4, respectively, and incubated at $37{ }^{\circ} \mathrm{C}$ for $1 \mathrm{~h}$. Diluted secondary antibodies (FITC-conjugated goat anti-rabbit IgG) were then added to all groups and plates were incubated at $37^{\circ} \mathrm{C}$ for $1 \mathrm{~h}$. 


\section{Detection of the transcription of NSP4 and VP7 genes in vivo}

Six-week-old mice were orally inoculated with $1 \times 10^{9}$ CFU of $\chi 4550$ (pVAXD-asd-NSP4-VP7). Three days post immunisation, Payer's patches were removed from three mice and pooled. Cellular RNA was isolated from homogenised Payer's patches with Trizol (TaKaRa, Dalian) according to the manufacturer's instructions. The transcripts of PRV NSP4 and VP7 genes in Payer's patches were analysed by means of reverse transcription (RT) PCR using specific primers $\mathrm{S} 1$ and S2 described earlier (Yang et al. 2009).

\section{Stability and safety of recombinant attenuated Salmonella typhimurium}

A single bacterial colony of the selected recombinant $\chi 4550$ (pVAXD-asd-NSP4-VP7) strain was inoculated into antibiotic-free Luria-Bertani (LB) liquid medium and incubated at $37{ }^{\circ} \mathrm{C}, 200 \mathrm{r} / \mathrm{min}$ until the optical density of the culture reached approximately 0.6 at $600 \mathrm{~nm}$. We then inoculated the bacterium (1:50) into antibiotic-free LB liquid medium and incubated samples at $37{ }^{\circ} \mathrm{C}, 200 \mathrm{r} / \mathrm{min}$. Over the next 18 hours, at three-hour intervals, the bacterium was transferred from the LB liquid medium onto LB agar medium and identified by RT-PCR with specific primer S2. A gavage needle was used to inoculate six-week-old BALB/c mice (groups of three) intragastrically with $\chi 4550$ (pVAXD-asdNSP4-VP7) at a dosage of $1 \times 10^{8} \mathrm{CFU}, 1 \times 10^{9} \mathrm{CFU}$ and $5 \times 10^{9}$ CFU. They were boosted with the same dosages two weeks later. At the same time, the control group was given PBS at the same dosages. The health of the mice was observed every day for 30 days.

\section{Inoculation of mice}

Eight-week-old BALB/c mice were randomly assigned to six groups of 15 each:

- Group A: PBS (pH 7.2)

- Group B: $\chi 4550$ (pVAX1-asd)

- Group C: $\chi 4550$ (pVAX1-asd-NSP4)

- Group D: $\chi 4550$ (pVAX1-asd-VP7)

- Group E: $\chi 4550$ (pVAXD-asd-NSP4-VP7)

- Group F: $\chi 4550$ (pVAX1-asd-NS4)+ $\chi 4550$ (pVAX1-asdVP7).

On day 1 mice of groups B, C, D and E were orally immunised with $0.2 \mathrm{~mL}$ Salmonella suspension $\left(1 \times 10^{9} \mathrm{CFU}\right)$ and boosted with the same dosage twice at two-week intervals. The coimmunised group (F) received the same dosage of two equally mixed Salmonella suspensions $\left(1 \times 10^{9} \mathrm{CFU}\right)$ on days 1,14 and 28 . The control group (A) received $0.2 \mathrm{~mL}$ PBS on days 1,14 and 28 .

\section{Collection and analysis of samples}

Three mice from each group were sacrificed for sera and intestinal lavage on days 1, 14 and 28. Blood was collected from the submaxillary sinuses of the mice and sera were prepared and stored at $-20^{\circ} \mathrm{C}$ until use. The intestinal fluid of each mouse was prepared by dicing the entire small intestine, excluding fat and the pancreas, and triturating it in $5 \mathrm{~mL}$ PBS containing $1 \mathrm{mmol} / \mathrm{L}$ phenylmethylsulfonyl fluoride (PMSF). To remove cellular debris, samples were centrifuged at $12000 \times g$ for $30 \mathrm{~min}$. The supernatant was collected as resultant intestinal lavage and stored at $-20{ }^{\circ} \mathrm{C}$ until use.

For measurement of rotavirus serum IgG and intestinal IgA levels in immunised mice, an indirect ELISA was used as described previously (Yang et al. 2009). Data of serum $\operatorname{IgG}$ and intestinal $\operatorname{Ig} \mathrm{A}$ titres were analysed using one-way analysis of variance. Significant differences were found amongst all immunisation groups ( $p \leq 0.05)$.

\section{Measurement of interferon- $\gamma$ production}

Spleens of three mice from group E were removed aseptically and pooled into $10 \mathrm{~mL}$ RPMI1640 medium 14 days after the third immunisation. Splenocytes were isolated by using a cell constrainer and centrifuged at $1000 \mathrm{rpm}$ for $10 \mathrm{~min}$ at room temperature. The cell pellet was re-suspended in $10 \mathrm{~mL}$ of the mentioned medium and the concentration of cells was adjusted to $5 \times 10^{6}$ cells $/ \mathrm{mL}$. An aliquote of $2 \mathrm{~mL}$ of this cell suspension and $20 \mu \mathrm{g}$ of stimulant protein NSP4 and VP7 equivalent admixture were added to each well of a 24-well pate. Plates were incubated in $\mathrm{CO}_{2}$ at $37{ }^{\circ} \mathrm{C}$ for $72 \mathrm{~h}$. The splenocyte culture supernatants were collected for interferon- $\gamma$ (IFN- $\gamma$ ) analysis using an IFN- $\gamma$ ELISA kit according to the manufacturer's instructions (Fangcheng Biotech, Beijing).

\section{Ethical considerations}

Animals were handled humanely during sample collection under the supervision of veterinarians and according to procedures complying with Chinese laws.

\section{Results}

\section{Reforming eukaryotic expression vector pVAX1-asd and pVAXD-asd}

The asd segments with flat ends were ligated into plasmid pVAX1 and pVAXD and then heat-shock transformated into

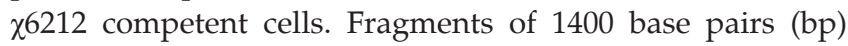
were amplified by RT-PCR from a single bacterial colony, indicating that the vectors $\mathrm{pVAX1}$-asd and $\mathrm{pVAXD}$-asd were reformed successfully (Figure 1).

\section{Construction of the transient expression of vaccine plasmid pVAX1-asd-VP7, pVAX1-asd- NSP4, pVAXD-asd-NSP4-VP7}

A fragment of $300 \mathrm{bp}$ was amplified by RT-PCR from the PRV OSU strain. Sequence analysis showed that the amplified sequence was $267 \mathrm{bp}$ in length and encoded 89 amino acids. The result of sequence alignment showed that the predicted amino acid sequence exhibited 97.8\% homology with the sequence of the NSP4 gene fragment (87-175) of the PRV OSU strain. The NSP4 gene fragment was inserted into the pVAX1asd and pVAXD-asd vectors. Another 1000-bp fragment was 


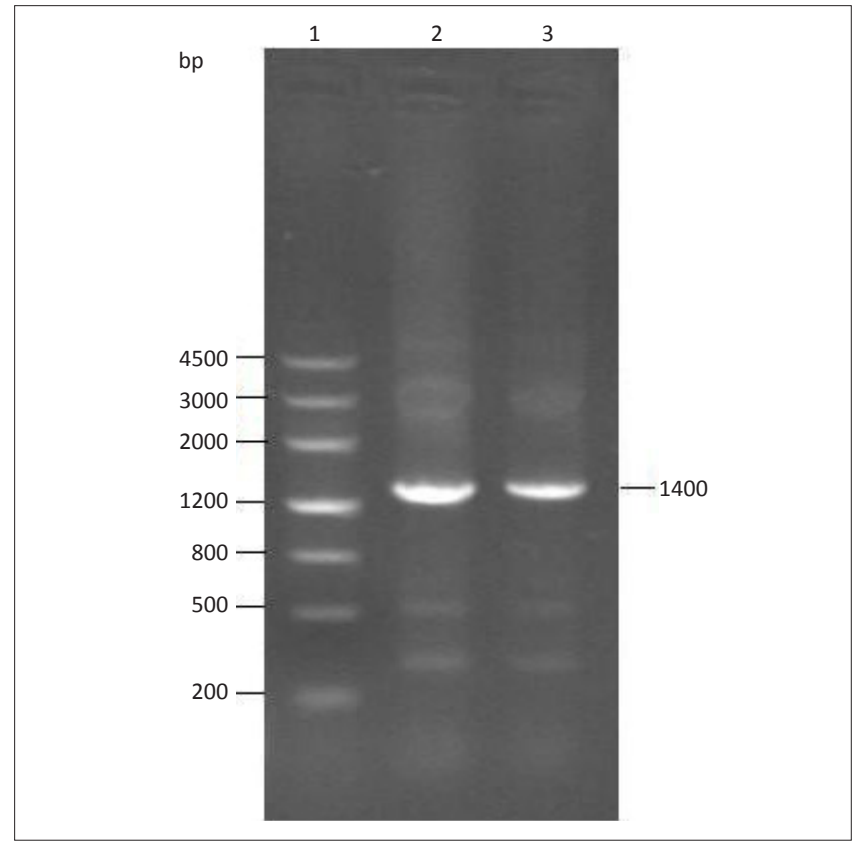

Column 1, DNA marker III; Column 2, products of recombinant pVAX1-asd coenobium polymerase chain reaction identification; Column 3 , products of recombinant pVAXD-asd coenobium polymerase chain reaction identification; bp, base pairs.

FIGURE 1: Identification electrophoresis of recombinant pVAX1-asd and pVAXD-asd with coenobium polymerase chain reaction.

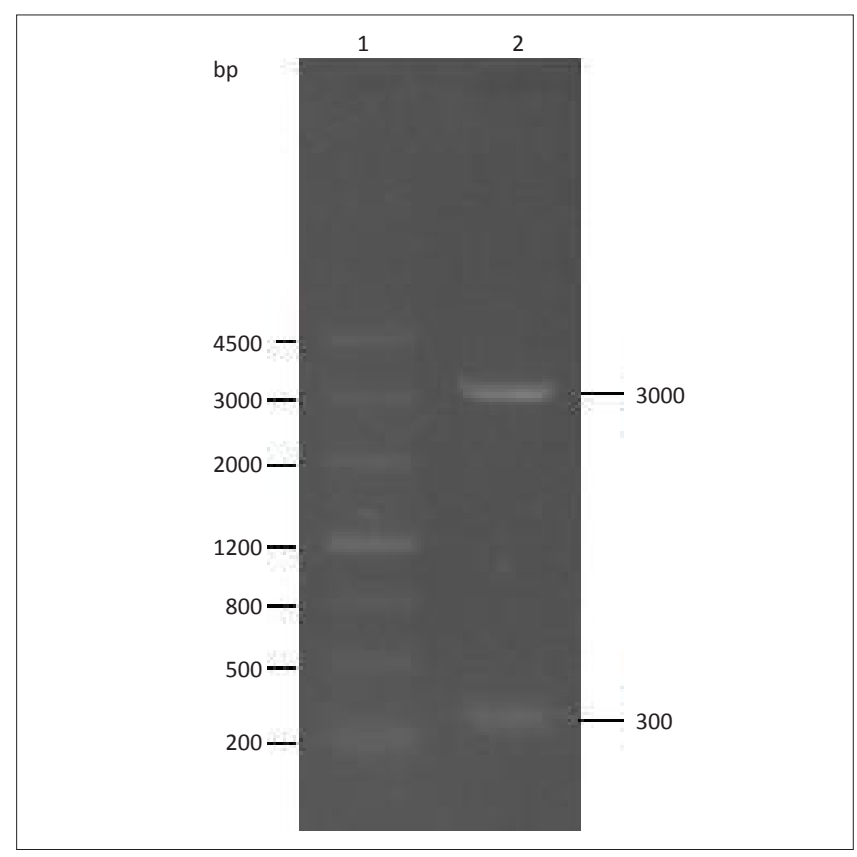

Column 1, DNA marker III; Column 2, pVAX1-asd-NSP4 digested with Pstl and Xhol; bp, base pairs.

FIGURE 2: Identification electrophoresis of recombinant pVAX1-asd-NSP4 with restriction enzyme.

amplified by RT-PCR from the PRV OSU strain. Sequence analysis showed that the amplified sequence was $987 \mathrm{bp}$ in length and encoded 326 amino acids. The result of sequence alignment showed that the predicted amino acid sequence exhibited $99.4 \%$ homology with the sequence of the VP7 gene fragment of the PRV OSU strain. The VP7 gene fragment was inserted into the $\mathrm{pVAX1-asd}$ and $\mathrm{pVAXD}$-asd-NSP4 vectors. The recombinant plasmids pVAX1-asd-NSP4, pVAX1-asd-

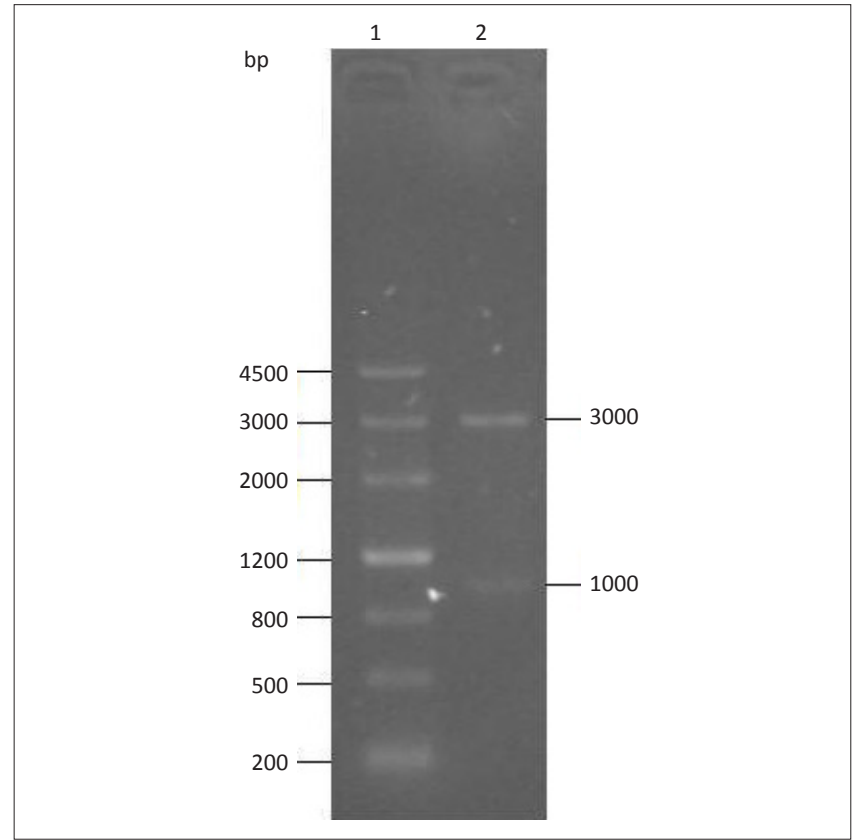

Column 1, DNA marker III; Column 2, pVAX1-asd-VP7 digested with Nhel and Kpnl; bp, base pairs.

FIGURE 3: Identification electrophoresis of recombinant pVAX1-asd-VP7 with restriction enzyme.

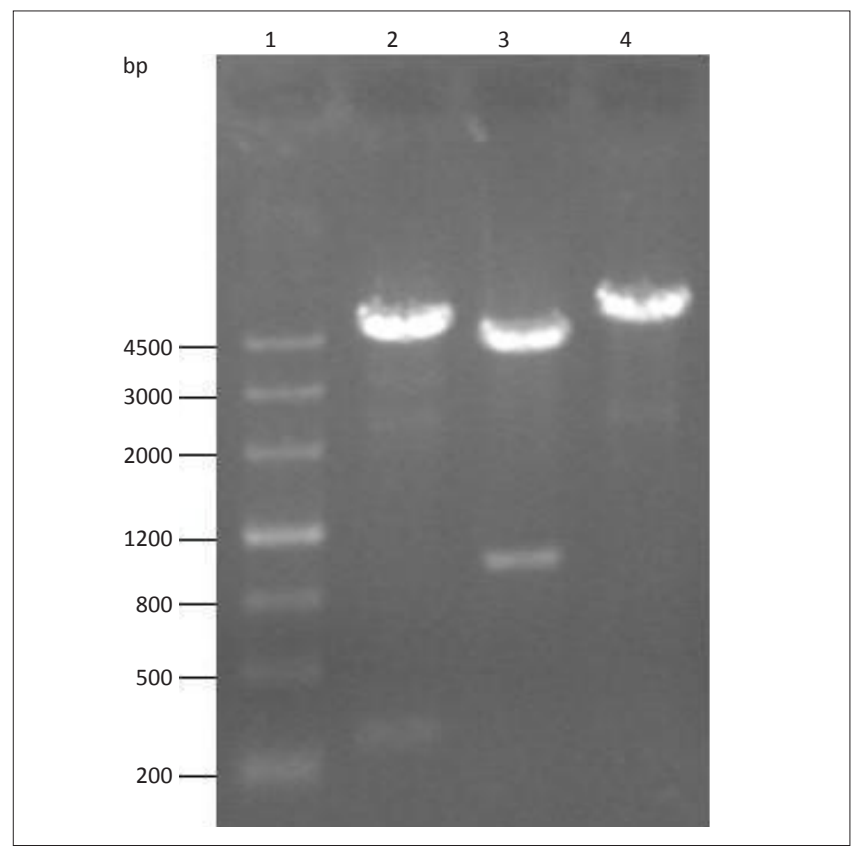

Column 1, DNA marker III; Column 2, pVAXD-asd-NSP4-VP7 digested with Pstl and Xhol; Column 3, pVAXD-asd-NSP4-VP7 digested with Nhel and Kpnl; Column 4, pVAXD-asd-NSP4VP7 digested with Pstl; bp, base pairs.

FIGURE 4: Identification electrophoresis of recombinant pVAXD-asd-NSP4-VP7 with restriction enzyme.

VP7 and pVAXD-asd-NSP4-VP7 were electroporated into $S$. typhimurium $\chi 4550$ strain. They were subsequently detected by enzyme restriction analysis (Figures 2, 3 and 4).

\section{Transient expression of recombinant plasmids in COS-7 cells}

The expression of recombinant plasmids pVAX1-asdVP7, pVAX1-asd-VP7 and pVAXD-asd-NSP4-VP7 was 

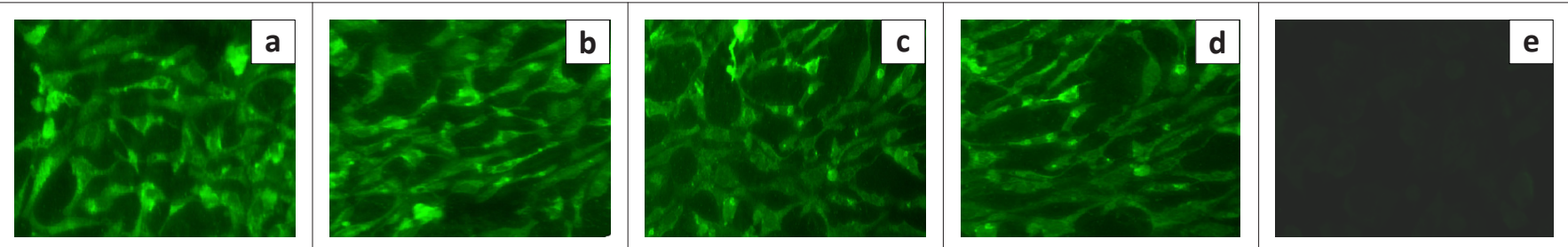

FIGURE 5: Indirect immunofluorescence detection of the expression of recombinant plasmids in COS-7 cells. (a) Indirect immunofluorescence result of COS-7 cells transfected with pVAX1-asd-VP7; (b) Indirect immunofluorescence result of COS-7 cells transfected with pVAX1-asd-NSP4; (c) Indirect immunofluorescence result of COS7 cells transfected with pVAXD-asd-NSP4-VP7, by using anti-NSP4 serum; (d) Indirect immunofluorescence result of COS-7 cells transfected with pVAXD-asd-NSP4-VP7, by using anti-VP7 serum and (e) Indirect immunofluorescence result of COS-7 cells transfected with pVAX1-asd, by using anti-VP7 serum.

demonstrated by an indirect immunofluorescence assay. Cytoplasmic fluorescence was observed in COS-7 cells transfected with all the recombinant plasmids, but not with the parental vector pVAX1 (Yang et al. 2009) (Figure 5).

\section{Transcription of recombinant plasmids in vivo using attenuated Salmonella typhimurium as transgenic vehicle}

Three days after intragastrically inoculating mice with attenuated S. typhimurium $\chi 4550$ (pVAXD-asd-NSP4-VP7), Payer's patches were removed from three mice and used for RNA extraction. Payer's patches are not only the main colonisation site of attenuated S. typhimurium but also an important immunologically relevant site in the context of mucosal responsiveness. Two fragments of $300 \mathrm{bp}$ and $1000 \mathrm{bp}$, respectively, were amplified by RT-PCR (Figures 6 and 7).

\section{Stability and safety of recombinant attenuated Salmonella typhimurium}

Selected single bacterial colonies of recombinant $\chi 4550$ (pVAXD-asd-NSP4-VP7) were inoculated into antibiotic-free LB medium. The recombinant plasmids were subsequently identified by RT-PCR for 18 hours at three-hour intervals. The positive ratio reached $100 \%$, showing that the recombinant plasmid was of high stability. BALB/c mice that had been inoculated orally with $\chi 4550$ (pVAXD-asd-NSP4-VP7) at different dosages were used for safety analysis. No adverse reactions were observed during the observation period; the diet, stools and health of the mice were all normal.

\section{Production of serum anti-rotavirus IgG antibodies}

Following oral immunisation with all recombinant S. typhimurium vaccines, all mice developed detectable serum IgG titres against rotavirus. Serum anti-rotavirus IgG was measured by indirect ELISA using a 1:100 dilution of immunised mouse serum. As shown in Figure 8, the anti-rotavirus IgG was produced fast and appeared 28 days post inoculation. In group B and C, we could infer that the antibody level of VP7 expression was significantly higher than NSP4 expression $(p<0.05)$. In addition, the coexpression of recombinant $S$. typhimurium $\chi 4550$ (pVAXDasd-NSP4-VP7) produced the highest antibody level in group E, which was somewhat similar to that of group D and group F. No specific anti-rotavirus antibodies were observed in serum samples of group A (PBS) or group B ( $\chi 4550$ [pVAX-

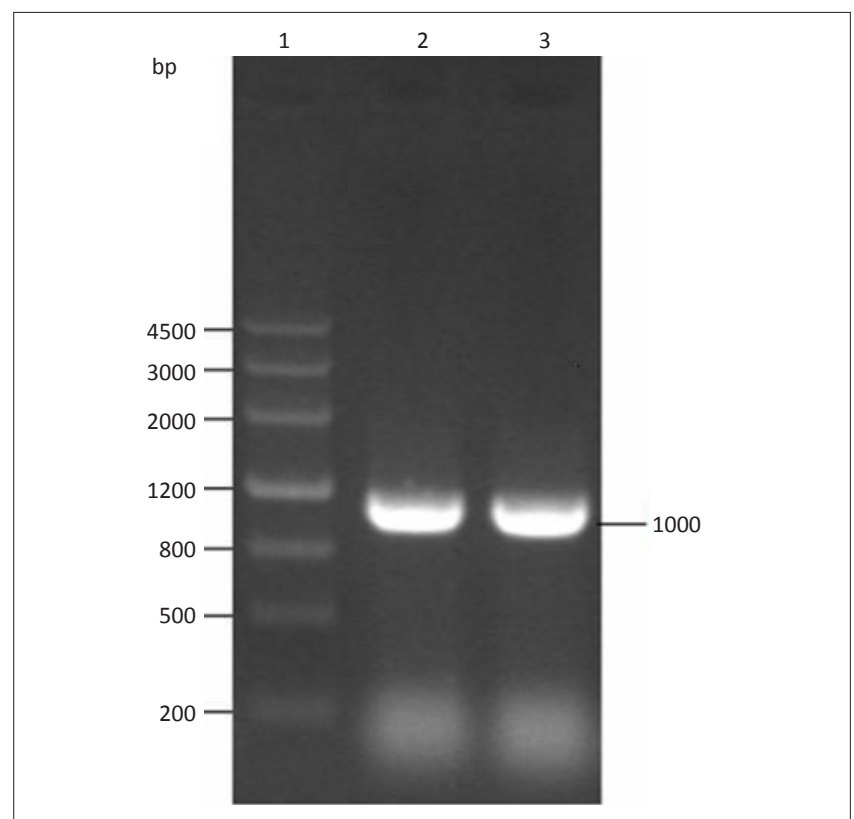

Column 1, DNA marker III; Columns 2 and 3, transcription of VP7 gene in vivo after mice were immunised orally with $x 4550$ (pVAXD-asd-NSP4-VP7).

FIGURE 6: Detection of the transcription of the VP7 gene in vivo after mice were immunised orally with recombinant Salmonella typhimurium (reverse transcription polymerase chain reaction).

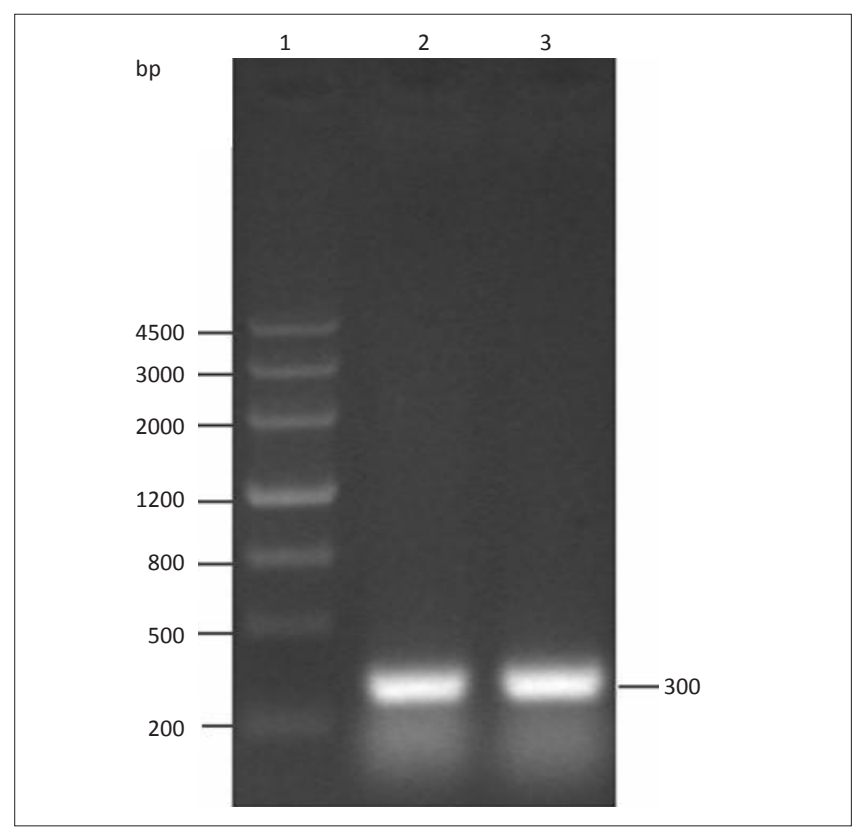

Column 1, DNA marker III; Columns 2 and 3, detection of the transcription of the NSP4 gene in vivo after mice were immunised orally with $\chi 4550$ (pVAXD-asd-NSP4-VP7).

FIGURE 7: Detection of the transcription of the NSP4 gene in vivo after mice were immunised orally with recombinant Salmonella typhimurium (reverse transcription polymerase chain reaction). 
asd]). The experiment demonstrated that the co-expression $S$. typhimurium vaccine may considerably enhance the humoural immune response.

\section{Production of intestinal lavage anti-rotavirus IgA antibodies}

The ability of the recombinant $S$. typhimurium vaccine to induce a mucosal immune response was determined by detecting the level of IgA antibodies to rotavirus in intestinal lavage samples. As shown in Figure 9, the anti-rotavirus IgA was produced fast and first appeared after 14 days. A oneway analysis of variance indicated that there was not much difference amongst groups C, D, E and F, which received $\chi 4550$ (pVAX1-asd-NSP4), $\chi 4550$ (pVAXD-asd -VP7), $\chi 4550$ (pVAXD-asd-NSP4-VP7), $\chi 4550$ (pVAX1-asd-NSP4) and $\chi 4550$ (pVAXD-asd -VP7), respectively. Up to day 28 post inoculation, there were significant differences amongst each group. The IgG antibody level of co-expression group E was the highest, that of group B and C was similarly low and for co-immunised group $\mathrm{F}$ the antibody level was notably higher than that of group C and D, but somewhat lower than for group E. No specific anti-rotavirus antibodies were observed in the intestinal lavage sample of group A (PBS) or group B ( $\chi 4550$ [pVAX-asd]).

\section{Detection of interferon- $\gamma$ secretion by splenocytes}

The secretion of IFN- $\gamma$ by splenocytes in the supernatant of the cultures was detected by ELISA. A total of $1097.693 \mathrm{pg} / \mathrm{mL}$ IFN- $\gamma$ was secreted by group E, which was much higher than that of the control group (group B) at $781.7125 \mathrm{pg} / \mathrm{mL}$. This indicated that the immunity of mice was considerably increased post inoculation with the $\chi 4550$ (pVAXD-asdNSP4-VP7) vaccine.

\section{Trustworthiness}

We declare that the experiments were conducted in a reliable, authentic and valid manner.

\section{Discussion}

This experiment demonstrated that a bicistronic DNA vaccine that encodes the PRV VP7 protein and the C-terminal of the NSP4 protein simultaneously and is delivered by an attenuated S. typhimurium $\chi 4550$ strain, was a potent vaccine, which induced a significantly higher level of serum IgG, mucosal IgA and splenocyte IFN- $\gamma$ than in the mice immunised with a single-expression vaccine.

DNA immunisation is a considerable vaccination strategy, which has many desirable characteristics for an ideal vaccine, including induction of broad immune responses and longlasting immunity against diseases. In addition, oral delivery of plasmid DNA vaccines with bacteria as carriers is more efficacious than traditional DNA vaccines. Compared 'to immunization with naked plasmid DNA, no further plasmid amplification and purification steps are needed, thereby

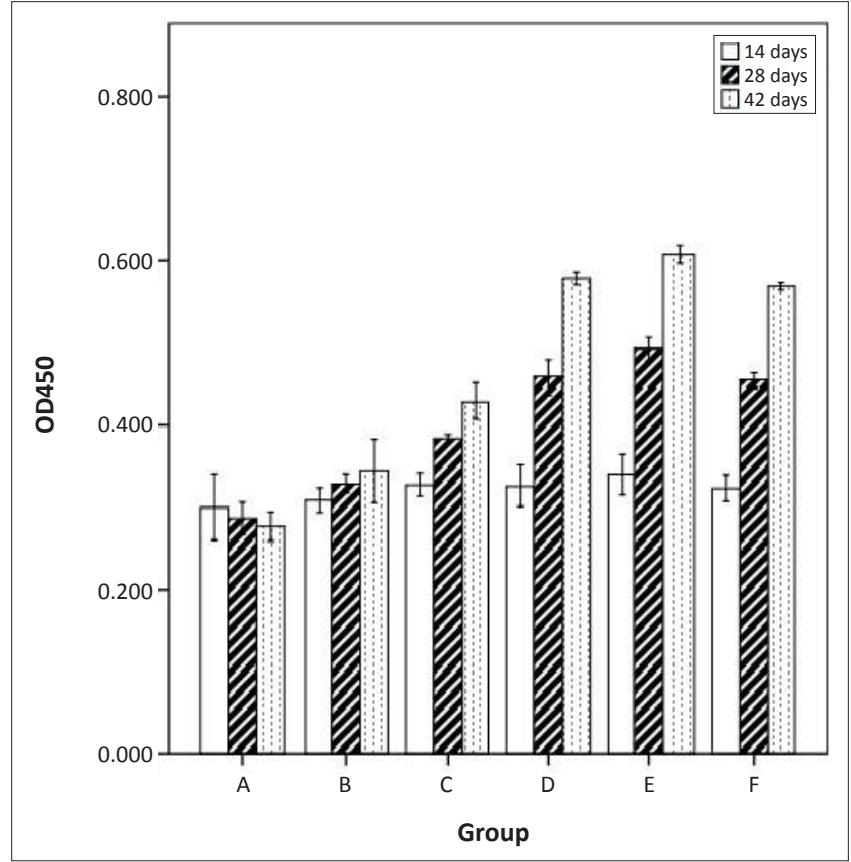

OD450, optical density at $450 \mathrm{~nm}$.

FIGURE 8: Indirect enzyme-linked immunosorbent assay analysis of the antirotavirus IgG antibody in murine serum after immunisation with different vaccines.

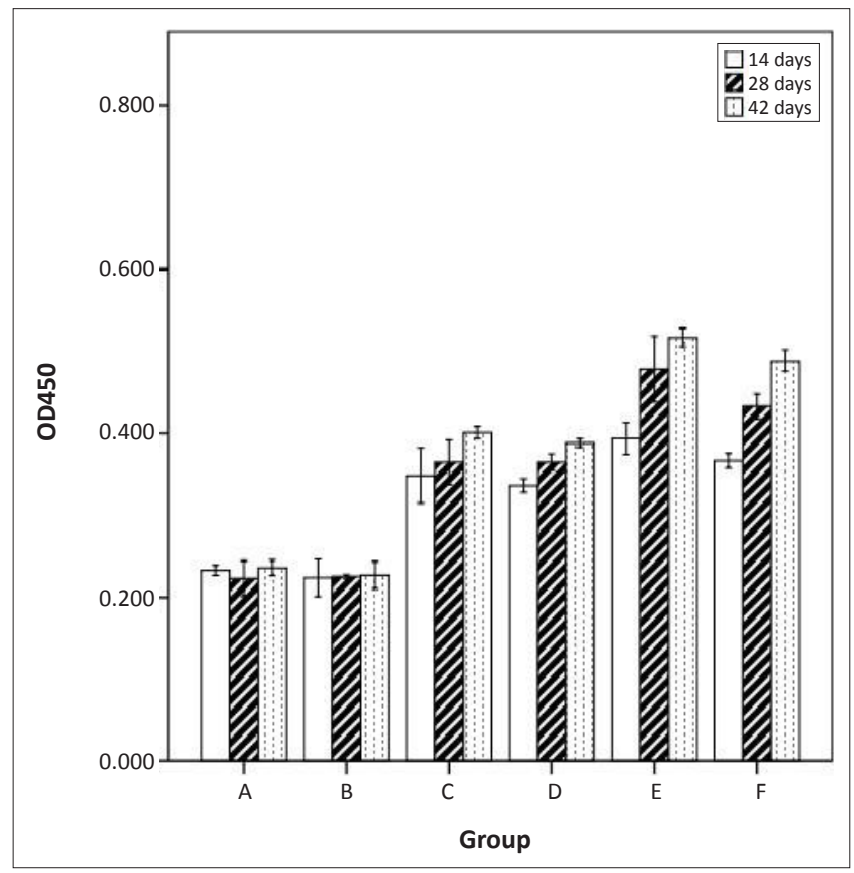

OD450, optical density at $450 \mathrm{~nm}$.

FIGURE 9: Indirect enzyme-linked immunosorbent assay analysis of antirotavirus IgA antibody in murine serum after immunisation with different vaccines.

reducing cost and labour extensively' (Schoen et al. 2004). Oral immunisation with a rotavirus DNA vaccine was first demonstrated by Chen et al. (1997). Yang et al. (2001) reported that intramuscular immunisation of mice with VP6 DNA vaccines induced high levels of VP6-specific serum IgG and IgA antibodies but not fecal IgA antibodies. Our study provided evidence that attenuated $S$. typhimurium strain $\chi 4550$ could be used as an oral delivery vector for PRV DNA 
vaccines. The target gene could be expressed both in vitro and in vivo to develop a specific humoural, mucosal and cellular immune response against PRV.

Live bacteria as DNA vaccine vectors offer many potential advantages in contrast to inactivated or attenuated vaccines. They are able to deliver multiple antigens safely with easy oral immunisation. Bacteria naturally contain immunostimulatory molecules such as lipopolysaccharides, which can function as an adjuvant to provoke immune responses (Pasetti, Levine \& Sztein 2003). Besides, Salmonella carrier strains have been shown to escape from the primary vacuole of the phagosome and transfer plasmids effectively to mammalian host cells in vivo (Schoen et al. 2004). Live Salmonella vectors have also been shown to induce a systemic immune response, including humoural, cellular and mucosal immunity against pathogen infection (Lintermans \& Greve 1995).

Safety is a prerequisite for plasmid DNA vaccines with live bacteria as vectors. In this study, we reconstructed the new plasmid by removing the kanamycin resistance gene and replaced it with the asd gene using a balanced lethal system. RT-PCR was used to ensure that the plasmids, pVAX1-asd and pVAXD-asd, were reconstructed successfully. The asd gene is a diaminopimelic acid (DAP) biosynthetic gene, which is an important component of the cell wall of Gramnegative bacteria. As bacterial amino acids are not found in eukaryotes, no Salmonella could survive without DAP. An S. typhimurium $\chi 4550$ strain without an asd gene was chosen as the plasmid DNA carrier as it was the only strain in which reconstructed plasmids could survive. During the experiment, the diet, stools and health of mice were all normal and no adverse reactions were observed. All the results suggested that the reconstructed S. typhimurium $\chi 4550$ strain vaccine was safe.

The structural protein VP7 of a rotavirus constitutes the outer shell of the virion and elicits the production of distinct neutralising antibodies in the host. It is thought to play an important role in protecting rotavirus-induced diarrhoea. In previous research a murine model was constructed to express the rotavirus VP7 protein with a transmissible gastroenteritis virus vector to immunise and provide protection against infections (Ribes et al. 2011). 'Rotavirus-specific antibodies were found only after immunization by the intraperitoneal route. Partial protection against rotavirus-induced diarrhoea was achieved in suckling BALB/c mice born to dams immunized with the recombinant virus' (Ribes et al. 2011) when they were orally challenged with the homotypic rotavirus. According to this study, improving the immunity of VP7 was stringently demanded. Kavanagh et al. (2010) also demonstrated that the NSP4 protein of a rotavirus has mucosal adjuvant properties, because it is a multifunctional protein that functions in rotavirus morphogenesis and pathogenesis and was the first viral enterotoxin to be described. This discovery correlates with the observation that inclusion of specific adjuvants in vaccines can modify the presentation modality of antigens to the immune system and improve the induction of the immune response over that induced by the same antigen given alone (Buonaguro, Tornesello \& Buonaguro 2009 in Kavanagh et al. 2010). For these reasons, we reconstructed a bicistronic DNA vaccine harbouring the VP7 and NSP4 proteins synchronously. Both serum IgG and mucosal $\operatorname{IgA}$ antibody response against the rotavirus were detected post inoculation with the DNA vaccine. The vaccine expressing only the NSP4 protein elicited a weak anti-rotavirus $\operatorname{IgG}$ and $\operatorname{IgA}$ antibody response. However, the vaccine expressing only the VP7 protein induced a significantly stronger response, similar to that induced by co-immunised NSP4 and VP7 proteins. The bicistronic DNA vaccine co-expressing the VP7 and NSP4 proteins induced the highest IgG and IgA antibody levels. Thus, we concluded that the immunogenicity of a rotavirus DNA vaccine could be improved by co-expressing the VP7 and NSP4 proteins.

This study was the first to construct a eukaryotic bicistronic plasmid DNA vaccine harboured by the prokaryotic expressing S. typhimurium $\chi 4550$ strain and elicited desirable humoural and mucosal immune response against rotavirus. Further experiments are needed to elucidate the immunologic mechanism of this recombinant DNA vaccine.

\section{Conclusion}

We described the construction of a bicistronic plasmid DNA vaccine against porcine rotavirus, which carries the NSP4 and VP7 genes simultaneously. BALB/c mice were orally immunised with an attenuated S. typhimurium $\chi 4550$ strain expressing the vaccine. Significant levels of serum IgG, intestinal IgA and IFN- $\gamma$ were induced by the reconstructed bicistronic DNA vaccine, suggesting that it may be a promising vaccine candidate against porcine rotavirus.

\section{Acknowledgements}

We thank the Science and Technology support programme, Sichuan Province for integration and demonstration of the key technology research on modern pig industry.

\section{Competing interests}

The authors declare that they have no financial or personal relationship(s) that may have inappropriately influenced them in writing this article.

\section{Authors' contributions}

S.C. (Sichuan Agricultural University) was the project leader. T.C. (Sichuan Agricultural University) and J.X. (Sichuan Agricultural University) were responsible for experiment and project design and performed most of the experiments. X.W. (Sichuan Agricultural University) and X.H. (Sichuan Agricultural University) made conceptual contributions, Y.W. (Sichuan Rural Science and Technology Development Centre) prepared the samples, Y.H. (Sichuan Agricultural University), X.M. (Sichuan Agricultural University) and Z.H. (Sichuan Agricultural University) performed some 
of the experiments. Q.Z. (Sichuan Agricultural University) performed the calculations and guided the writing of the article.

\section{References}

Ball, J.M., Tian, P., Zeng, C.Q.Y, Morris, A.P. \& Estes, M.K., 1996, 'Age-dependen diarrhea induced by a rotaviral nonstructural glycoprotein', Science 272, 101-104 $\mathrm{http}: / / \mathrm{dx}$.doi.org/10.1126/science.272.5258.101, PMid:8600515

Ball, J.M., Mitchell, D.M., Gibbons, T.F. \& Parr, R.D., 2005, 'Rotavirus NSP4: A multifunctional viral enterotoxin', Viral Immunology 18, 27-40. http://dx.doi org/10.1089/vim.2005.18.27, PMid:15802952

Buonaguro, F.M., Tornesello, M.L. \& Buonaguro, L., 2009, 'Virus-like particle vaccines and adjuvants: The HPV paradigm', Expert Review of Vaccines 8, 1379-1398. $\mathrm{http}: / / \mathrm{dx}$.doi.org/10.1586/erv.09.81, PMid:19803760

Chen, S.C., Fynan, E.F., Robinson, H.L., Lu, S., Greenberg, H.B., Santoro, J.C. et al., 1997, 'Protective immunity induced by rotavirus DNA vaccines', Vaccine 15, 899902. http://dx.doi.org/10.1016/S0264-410X(96)00272-1

Chen, S.C., Jones, D.H., Fynan, E.F., Farrar, G.H., Clegg, J.C.S, Greenberg, H.B. et al., 1998, 'Protective immunity induced by oral immunization with a rotavirus DNA vaccine encapsulated in microparticles', Journal of Virology 72, 5757-5761. PMid:9621034

Coste, A., Sirard, J.C., Johansen, K., Cohen, J. \& Kraehenbuhl, J.P., 2000, 'Nasal immunization of mice with virus-like particles protects offspring against rotavirus diarrhea', Journal of Virology 74, 8966-8971. http://dx.doi.org/10.1128/ JVI.74.19.8966-8971.2000, PMid:10982340

Estes, M.K., 2001, 'Rotaviruses and their replication', in D.M. Knipe, P.M. Howley, D.E. Griffin, R.A. Lamb, M.A. Martin, B. Roizman et al. (eds.), Fields Virology, 4th edn., pp. 1747-1785, Lippincott Williams \& Wilkins, Philadelphia.

Gebert, A., 1997, 'The role of $M$ cells in the protection of mucosal membranes', Histochemistry and Cell Biology 108, 455-470. http://dx.doi.org/10.1007/ s004180050186, PMid:9450628

Glass, R.I., Parashar, U.D., Bresee, J.S., Turcios, R., Fischer, T.K., Widdowson, M.A. et al., 2006, 'Rotavirus vaccines: Development, current prospects and future challenges', Lancet 368, 323-32. http://dx.doi.org/10.1016/S0140-6736(06)68815-6

Gonzalez, A.M., Nguyen, T.V., Azevedo, M.S.P., Jeong, K., Agarib, F., losef, C. et al., 2004, 'Antibody responses to human rotavirus (HRV) in gnotobiotic pigs following a new prime/boost vaccine strategy using oral attenuated HRV priming and intranasal VP2/6 rotavirus-like particle (VLP) boosting with ISCOM', Clinical \& Experimental Immunology 135, 361-372. http://dx.doi.org/10.1111/j.13652249.2004.02395.x, PMid:15008967

Herrmann, J.E., Chen, S.C., Jones, D.H., Tinsley-Bown, A., Fynan, E.F., Greenberg, H.B. et al., 1999, 'Immune responses and protection obtained by oral immunization with rotavirus VP4 and VP7 DNA vaccines encapsulated in microparticles', Virology 259, 148-153. http://dx.doi.org/10.1006/viro.1999.9751, PMid:10364499

Hoshino, Y., Saif, L.J., Sereno, M.M., Chanock, R.M. \& Kapikian, A.Z., 1988, 'Infection immunity of piglets to either VP3 or VP7 outer capsid protein confers resistance to challenge with a virulent rotavirus bearing the corresponding antigen', Journal of Virology 62, 744-748. PMid:2828669
Kavanagh, O.V., Ajami, N.A., Cheng, E., Ciarlet, M., Guerrero, R.A., Zeng, C.Q.Y. et al., 2010, 'Rotavirus enterotoxin NSP4 has mucosal adjuvant properties', Vaccine 28, 3106-3111. http://dx.doi.org/10.1016/j.vaccine.2010.02.063, PMid:20197138

Lintermans, P. \& Greve, H.D., 1995, 'Live bacterial vectors for mucosal immunization', Advanced Drug Delivery Reviews 18, 73-89. http://dx.doi.org/10.1016/0169409X(95)00051-8

Matsui, S.M., Offit, P.A., Vo, P.T., Mackow, E.R., Benfield, D.A., Shaw, R.D. et al., 1989, 'Passive protection against rotavirus-induced diarrhea by monoclonal antibodies to the heterotypic neutralization domain of VP7 and the VP8 fragment of VP4', Journal of Clinical Microbiology 27, 780-782. PMid:2470774

O’Neal, C.M., Crawford, S.E., Estes, M.K. \& Conner, M.E., 1997, 'Rotavirus virus-like particles administered mucosally induce protective immunity', Journal of Virology particles administered mucosally in

Pasetti, M.F., Levine, M.M. \& Sztein, M.B., 2003, 'Animal models paving the way for clinical trials of attenuated Salmonella enterica serovar typhi live oral vaccines and live vectors', Vaccine 21, 401-418. http://dx.doi.org/10.1016/S0264410X(02)00472-3

Petrie, B.L., Estes, M.K. \& Graham, D.Y., 1983, 'Effects of tunicamycin on rotavirus morphogenesis and infectivity', Journal of Virology 46, 270-274. PMid:6298460

Ribes, J.M., Ortego, J., Ceriani, J., Montava, R., Enjuanes, L. \& Buesa, J., 2011 'Transmissible gastroenteritis virus (TGEV)-based vectors with engineered murine tropism express the rotavirus VP7 protein and immunize mice against murine tropism express the rotavirus VP7 protein and immunize mice against
rotavirus', Virology 410, 107-118. http://dx.doi.org/10.1016/j.virol.2010.10.036, rotavirus', Virology
PMid:21094967

Schoen, C., Stritzker, J., Goebel, W. \& Pilgrim, S., 2004, 'Bacteria as DNA vaccine carriers for genetic immunization', International Journal of Medical Microbiology 294, 319-335. http://dx.doi.org/10.1016/j.ijmm.2004.03.001, PMid:15532991

Song, X.F. \& Hao, Y., 2009, 'Adaptive evolution of rotavirus VP7 and NSP4 genes in different species', Computational Biology and Chemistry 33, 344-349. http:// dx.doi.org/10.1016/j.compbiolchem.2009.07.008, PMid:19665933

Ward, L.A., Rich, E.D. \& Besser, T.E., 1996, 'Role of maternally derived circulating antibodies in protection of neonatal swine against porcine group A rotavirus',
Journal of Infectious Diseases 174, 276-282. http://dx.doi.org/10.1093/ Journal of Infectious Diseases
infdis/174.2.276, PMid:8699055

Ward, R.L., Knowlton, D.R., Greenberg, H.B., Schiff, G.M. \& Bernstein, D.I., 1990, 'Serum-neutralizing antibody to VP4 and VP7 proteins in infants following vaccination with WC3 bovine rotavirus', Journal of Virology 64, 2687-2691. PMid:2159538

Ward, R.L., McNeal, M.M., Sander, D.S., Greenberg, H.B. \& Bernstein, D.I., 1993, Immunodominance of the VP4 neutralization protein of rotavirus in protective natural infections of young children', Journal of Virology 67, 464-468. PMid:8416378

Yang, H., Cao, S.J., Huang, X.B., Liu, J.W., Tang, Y. \& Wen, X.T., 2009, 'Intragastric administration of attenuated Salmonella typhimurium harbouring transmissible gastroenteritis virus (TGEV) DNA vaccine induced specific antibody production', Vaccine 27, 5035-5340. http://dx.doi.org/10.1016/j.vaccine.2009.06.050 PMid:19573642

Yang, K.J., Wang, S.X., Chang, K.O., Lu, S., Saif, L.J., Greenberg, H.B. et al., 2001, 'Immune responses and protection obtained with rotavirus VP6 DNA vaccines given by intramuscular injection', Vaccine 19, 3285-3291. http://dx.doi. given by intramuscular injection',
org/10.1016/S0264-410X(00)00543-0

Yuan, L., losef, C., Azevedo, M.S.P., Kim, Y., Qian, Y., Geyer, A. et al., 2001, 'Protective immunity and antibody-secreting cell responses elicited by combined oral attenuated Wa human rotavirus and intranasal Wa 2/6-VLPs with mutant Escherichia coli heat-labile toxin in gnotobiotic pigs', Journal of Virology $75,9229-$ 9238. http://dx.doi.org/10.1128/JVI.75.19.9229-9238.2001, PMid:11533185 